\title{
EFFECTS OF YIELD SURFACE SHAPE ON SHEET METAL FORMING SIMULATIONS
}

\author{
K.-C. LIAO ${ }^{1}$, J. PAN ${ }^{1, *}$ AND S. C. TANG ${ }^{2}$ \\ ${ }^{1}$ Mechanical Engineering and Applied Mechanics, The University of Michigan, Ann Arbor, MI 48109, U.S.A. \\ ${ }^{2}$ Ford Motor Company, Dearborn, MI 48121, U.S.A.
}

\begin{abstract}
Three phenomenological yield criteria are adopted to describe the plastic behaviour of sheet metals with normal plastic anisotropy. The sheet metals are assumed to be elastic-plastic, rate-sensitive and incompressible. A rate-sensitive thin shell finite element formulation based on the virtual work principle is derived for the three yield criteria. The effects of the yield surface shapes based on the three yield criteria with the same value of the plastic anisotropy parameter $R$ on the strain distribution and localization are investigated under a hemispherical punch stretching operation and a plane strain drawing operation. The results of the simulations show that the yield surface shape, in addition to the plastic anisotropy parameter $R$, controls the punch force, strain distribution and strain localization for the punch stretching operation. However, the yield surface shape does not affect the punch force and the strain distribution significantly for the plane strain drawing operation. (C) 1998 John Wiley \& Sons, Ltd.
\end{abstract}

Int. J. Numer. Meth. Engng., 41, 559-584 (1998)

KEY WORDS: sheet metal forming; plastic anisotropy; viscoplasticity; yield surface shape; thin shell finite element

\section{INTRODUCTION}

In sheet metal forming simulations, several finite element models such as membrane, solid and shell models are proposed. The membrane model, due to its simplicity, is frequently adopted to explain the necking and fracture behaviour in sheet forming processes (for example, see the works of Wang and Budiansky ${ }^{1}$ and Tang ${ }^{2}$ ). However, the membrane model is not appropriate where the bending of the sheet becomes significant, for example, in drawing operations (for example, see the works of Wang and Tang ${ }^{3}$ ). In general, the processes where the bending effect is significant are simulated by the solid or the shell model. For the solid model (for example, see the works of $\mathrm{Wifi}^{4}$ and Anderson ${ }^{5}$ ), more elements are needed to describe these shell-type structures to prevent numerical difficulty so that a large system of equations must be solved. The shell model (for example, see the works of Triantafyllidis and Samanta ${ }^{6}$ and Wang and Tang $^{3}$ ) is more effective than the solid model although integration in the thickness direction is still needed.

${ }^{*}$ Correspondence to: J. Pan, Mechanical Engineering, University of Michigan, Ann Arbor, MI 48109-2125, U.S.A. E-mail: jwo@umich.edu

Contract grant sponsor: National Science Foundation; Contract grant number: DDM-9102424

Contract grant sponsor: Ford Motor Company

CCC 0029-5981/98/030559-26\$17.50

Received 14 April 1997

(C) 1998 John Wiley \& Sons, Ltd. 
Sheet metals usually display not only normal anisotropy but also planar anisotropy. In sheet metal forming simulations, an average value of the plastic anisotropy parameter $R$, defined as the ratio of the transverse (width) plastic strain rate to the through-thickness plastic strain rate under in-plane uniaxial tensile loading, is used to characterize the normal anisotropy of the sheets with the assumption of planar isotropy. Many phenomenological yield criteria were proposed in the past, for example, see an excellent review by Hosford. ${ }^{7}$ The Hill quadratic yield criterion, ${ }^{8}$ which can be used to account for plastic orthotropy, has been widely used in numerical analyses for sheet forming operations (for example, see the works of Tang ${ }^{2}$ and Wang and Tang ${ }^{3}$ ). Hill ${ }^{9}$ proposed non-quadratic yield criteria in order to encompass the anomalous behaviour of some metals. In recent years, non-quadratic yield criteria have received much attention (for example, see the works of Wagoner et al. ${ }^{10}$ ). Note that neither Hill's quadratic yield criterion ${ }^{8}$ nor Hosford's non-quadratic yield criterion ${ }^{7}$ can encompass the so-called anomalous behaviour of some metals. More recently, Barlat et al. ${ }^{11}$ proposed a six-component yield criterion to take account for the planar anisotropy. They showed that the yield surface shapes based on the six-component yield criterion agree well with those based on a polycrystal model especially near the equal biaxial loading direction.

In order to investigate the effects of plastic anisotropy and yield surface shape on sheet metal stretchability, Chan ${ }^{12}$ calculated forming limit diagrams using the approach of MarciniakKuczynski ${ }^{13}$ based on the Hill quadratic yield criterion ${ }^{8}$ and the Drucker yield criteria ${ }^{14}$ with both the flow and deformation plasticity formulations. Chan concluded that the limit strain decreases with increasing value of $R$ in the positive minor strain region of the forming limit diagram but increases with increasing $R$ in the negative minor strain region and is independent of $R$ under plane strain conditions (the minor strain is 0 ). Barlat ${ }^{15}$ proposed a ratio of the plane strain flow stress to the equal biaxial flow stress as a convenient parameter to characterize the stretchability of sheet metals under in-plane biaxial loading conditions. He showed that the stretchability of sheet metals based on Hosford's yield criterion ${ }^{16}$ increases as the ratio increases using the MarciniakKuczynski approach. ${ }^{13}$ Zhou and Lian ${ }^{17}$ used two characteristic parameters corresponding to the yield surfaces based on several Hill's non-quadratic yield criteria ${ }^{9}$ to examine the effects of the yield surface shape on the bulging pressure as well as the stain distribution under hydrostatic bulging processes. The results of these research works indicate that the yield surface shape affects significantly the forming limit of anisotropic sheet metals.

Graf and Hosford ${ }^{18}$ investigated the effects of $R$ on forming limit using the Hill quadratic yield criterion $^{8}$ and the Hosford yield criterion. ${ }^{16}$ They found that when Hill's quadratic yield criterion is employed, $R$ has significant effects on forming limit. When the higher-order yield criterion of Hosford $^{16}$ is employed, $R$ has virtually no effects on forming limit. The research results again indicate that a single parameter $R$ is not enough to characterize the forming limit and the yield surface shape affects the forming limit of anisotropic sheet metals. Note that the theoretical forming limit diagram is obtained from a material element subjected to biaxial in-plane straining. Typical forming processes, however, involve distributed straining states as well as other complicated factors such as friction and bending. The synergetic effects of these complicated factors and the anisotropic factor $R$ are unknown. Therefore, further investigations of the effects of $R$ and the yield surface shape on the strain distribution and failure of typical forming processes are needed.

Hutchinson and Neale ${ }^{19}$ investigated the effects of the material strain rate sensitivity on forming limit for isotropic sheets under in-plane biaxial loading conditions. They showed that a small amount of strain rate sensitivity can significantly increase the limit strains when compared with those based on the corresponding rate-insensitive analysis. Neale and $\mathrm{Chater}^{20}$ examined the combined effects of strain rate sensitivity and plastic anisotropy on sheet necking using the 
Marciniak-Kuczynski approach. ${ }^{13}$ Neale and Chater found that strain rate sensitivity can cause necking retardation and alter the shapes of forming limit curves in the range between the equal biaxial tension and the plane strain tension. They also found that the yield surface curvature can be an important factor on strain localization. The results of the simulations of sheet stretching operations by Wang and Wenner ${ }^{21}$ using a membrane theory indicate that sheet metals with a large rate sensitivity display a rather uniform strain distribution.

In this paper, we include the effects of rate sensitivity into the shell finite element formulation of Wang and Tang ${ }^{3}$ based on the rate-sensitive membrane finite element formulation of Wang and Wenner. ${ }^{21}$ To take account for rate sensitivity, we adopt the formulation of Peirce et al. ${ }^{22}$ based on Taylor's series expansion of the rate-sensitive plastic flow stress-strain relation. We adopt three yield criteria, Hill's quadratic, ${ }^{8}$ Hill's non-quadratic, ${ }^{9}$ and the six-component yield criteria, ${ }^{11}$ for sheet metals with normal anisotropy to understand the effects of $R$ and the yield surface shape on hemispherical punch stretching and plane strain drawing operations where the bending effects must be included.

Here we present the paper in the following order. First, we present a Lagrangian description of axisymmetric and plane strain shell elements. Then we present the rate-sensitive constitutive relation for the physical components of the Cauchy stress rates and the true strain rates. A thin shell finite element formulation based on the virtual work principle is followed. In Section 3, the effects of $R$ and the yield surface shape based on the three yield criteria on the strain distribution and the punch force for sheet metals are investigated under the hemispherical punch stretching and plane strain draw operations. Conclusions are made in Section 4.

\section{FORMULATION}

\subsection{Kinematics for axisymmetric and plane strain shell elements}

We adopt a Lagrangian description of large deformation of a thin shell with reference to the undeformed flat configuration. Figure 1 shows the horizontal displacement $u$ and the vertical displacement $w$ of a material point on the reference middle surface of the undeformed configuration with the normal in the $\gamma$ direction. In the figure, a material point on the reference middle surface with a set of cylindrical co-ordinates $(\rho, \theta, 0)$ is displaced to $(\rho+u, \theta, w)$. The meridian and circumferential stretch ratios of the material point on the reference middle surface, denoted as $\lambda_{1}^{(0)}$ and $\lambda_{2}^{(0)}$, respectively, are expressed as

$$
\begin{aligned}
& \lambda_{1}^{(0)}=\left[\left(1+\frac{\partial u}{\partial \rho}\right)^{2}+\left(\frac{\partial w}{\partial \rho}\right)^{2}\right]^{1 / 2} \\
& \lambda_{2}^{(0)}=(\rho+u) / \rho
\end{aligned}
$$

We assume that the normal of the middle plane remains normal to the deformed middle plane. The meridian and circumferential stretch ratios, denoted as $\lambda_{1}$ and $\lambda_{2}$, respectively, at a normal distance $z$ away from the deformed middle surface are written as

$$
\begin{aligned}
& \lambda_{1}=\lambda_{1}^{(0)}\left(1+\kappa_{1} z\right) \\
& \lambda_{2}=\lambda_{2}^{(0)}\left(1+\kappa_{2} z\right)
\end{aligned}
$$




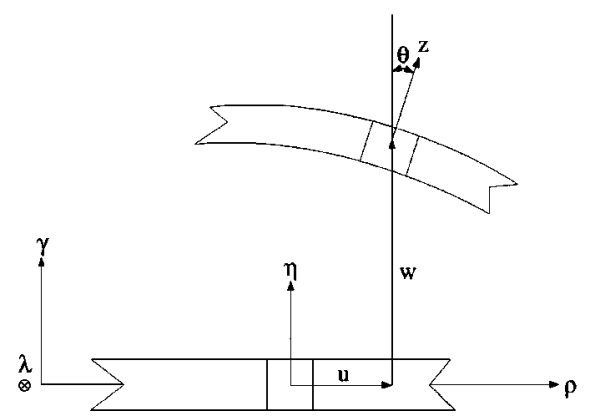

Figure 1. Initial and current configurations of a shell element

where $\kappa_{1}$ and $\kappa_{2}$ represent the principal curvatures in the meridian and circumferential directions, respectively,

$$
\begin{aligned}
& \kappa_{1}=\frac{\left[\frac{\partial w}{\partial \rho} \frac{\partial^{2} u}{\partial \rho^{2}}-\left(1+\frac{\partial u}{\partial \rho}\right) \frac{\partial^{2} w}{\partial \rho^{2}}\right]}{\left(\lambda_{1}^{(0)}\right)^{3}} \\
& \kappa_{2}=-\frac{\frac{\partial w}{\partial \rho}}{(\rho+u) \lambda_{1}^{(0)}}
\end{aligned}
$$

The logarithmic strain rates in the principal directions are defined as

$$
\dot{\varepsilon}_{\alpha}=\mathrm{d}\left(\ln \lambda_{\alpha}\right) / \mathrm{d} t=\dot{\lambda}_{\alpha}^{(0)} / \lambda_{\alpha}^{(0)}+\left(z \dot{\kappa}_{\alpha}+\kappa_{\alpha} \dot{z}\right) /\left(1+\kappa_{\alpha} z\right)
$$

where the Greek subscript ranges from 1 to 2 . It can be shown that the physical components of the true strain rates in the meridian and circumferential directions, $\dot{\varepsilon}_{r}$ and $\dot{\varepsilon}_{\theta}$, are equivalent to the logarithmic strain rates in the principal direction 1 and 2, respectively, under axisymmetric and plane strain loading conditions (for example, see the work of $\mathrm{Tang}^{2}$ ). The current distance $z$ of a material point from the reference surface can be related to its initial distance $\eta$ from the undeformed reference surface via the incompressibility as

$$
\frac{\mathrm{d} z}{\mathrm{~d} \eta}=\left(\lambda_{1} \lambda_{2}\right)^{-1}
$$

Integrating equation (8) with equations (3) and (4) gives $\eta$ as a function of $z$ as

$$
\eta=\lambda_{1}^{(0)} \lambda_{2}^{(0)}\left[z+\left(\kappa_{1}+\kappa_{2}\right) \frac{z^{2}}{2}+\kappa_{1} \kappa_{2} \frac{z^{3}}{3}\right]
$$

For a shell element under plane strain loading conditions, equations (1)-(9) are still valid except that $\lambda_{2}=1$ and $\kappa_{2}=0$.

\subsection{Constitutive relation}

We emphasize on the constitutive relation for sheet metal forming simulations in this paper. We assume that the sheet is subjected to plane stress loading conditions. The sheet metals are 
assumed to be elastic-plastic, rate-sensitive and incompressible. The sheet metals are assumed to have plastic planar isotropy with normal anisotropy. The physical components of the total strain rates in the meridian and circumferential directions are assumed to be the sum of the physical components of the elastic and the plastic strain rates in the corresponding directions as

$$
\begin{aligned}
& \dot{\varepsilon}_{r}=\dot{\varepsilon}_{r}^{\mathrm{e}}+\dot{\varepsilon}_{r}^{\mathrm{p}} \\
& \dot{\varepsilon}_{\theta}=\dot{\varepsilon}_{\theta}^{\mathrm{e}}+\dot{\varepsilon}_{\theta}^{\mathrm{p}}
\end{aligned}
$$

where $(\cdot)^{\mathrm{e}}$ and $(\cdot)^{\mathrm{p}}$ represent the elastic and plastic part of $(\cdot)$. The relation between the physical components of the elastic strain rates and the physical components of the Cauchy stress rates under plane stress conditions can be expressed in the matrix form as

$$
\left\{\begin{array}{c}
\dot{\varepsilon}_{r}^{\mathrm{e}} \\
\dot{\varepsilon}_{\theta}^{\mathrm{e}}
\end{array}\right\}=\frac{1}{E}\left[\begin{array}{cc}
1 & -v \\
-v & 1
\end{array}\right]\left\{\begin{array}{c}
\dot{\sigma}_{r} \\
\dot{\sigma}_{\theta}
\end{array}\right\}
$$

In equation (12), $E$ is Young's modulus, $v$ is Poisson's ratio, $\dot{\sigma}_{r}$ and $\dot{\sigma}_{\theta}$ are the physical components of the Cauchy stress rates in the meridian and circumferential directions, respectively. Here the sheet metals are assumed to have elastic isotropy. We define the elastic modulus matrix $\mathbf{L}$ as

$$
\mathbf{L}=\frac{1}{E}\left[\begin{array}{cc}
1 & -v \\
-v & 1
\end{array}\right]
$$

The plastic strain rates can be obtained by using the associated flow rule as

$$
\left\{\begin{array}{c}
\dot{\varepsilon}_{r}^{\mathrm{p}} \\
\dot{\varepsilon}_{\theta}^{\mathrm{p}}
\end{array}\right\}=\dot{\bar{\varepsilon}}^{\mathrm{p}}\left\{\begin{array}{l}
f_{r} \\
f_{\theta}
\end{array}\right\}
$$

where $\dot{\bar{\varepsilon}}^{\mathrm{p}}$ represents the effective plastic strain rate, and $f_{r}$ and $f_{\theta}$ can be obtained from the flow rule. Here we define the vector $\mathbf{f}$ as

$$
\mathbf{f}=\left\{\begin{array}{l}
f_{r} \\
f_{\theta}
\end{array}\right\}
$$

Note that $\mathbf{f}$ will be specified later for the various yield criteria.

Under plane stress conditions, the Hill quadratic yield criterion ${ }^{8}$ without shear stresses can be written as

$$
\Phi=\sigma_{r}^{2}+\sigma_{\theta}^{2}-\frac{2 R}{1+R} \sigma_{r} \sigma_{\theta}=\bar{\sigma}^{2}
$$

where $\Phi$ is the yield function, $\bar{\sigma}$ is the effective stress regarded as the yield stress under the inplane uniaxial tensile loading and $R$ is the plastic anisotropy parameter. The vector $\mathbf{f}$ defined in equation (15) can then be obtained by combining the equivalence of plastic work

$$
\sigma_{r} \dot{\varepsilon}_{r}^{\mathrm{p}}+\sigma_{\theta} \dot{\varepsilon}_{\theta}^{\mathrm{p}}=\bar{\sigma} \dot{\bar{\varepsilon}} \mathrm{p}
$$


and the associated flow rule as

$$
\begin{aligned}
\mathbf{f} & =\left\{\begin{array}{l}
\left(\bar{\sigma} \frac{\partial \Phi}{\partial \sigma_{r}}\right) /\left(\sigma_{r} \frac{\partial \Phi}{\partial \sigma_{r}}+\sigma_{\theta} \frac{\partial \Phi}{\partial \sigma_{\theta}}\right) \\
\left(\bar{\sigma} \frac{\partial \Phi}{\partial \sigma_{\theta}}\right) /\left(\sigma_{r} \frac{\partial \Phi}{\partial \sigma_{r}}+\sigma_{\theta} \frac{\partial \Phi}{\partial \sigma_{\theta}}\right)
\end{array}\right\} \\
& =\frac{1}{\bar{\sigma}}\left\{\begin{array}{c}
\sigma_{r}-\frac{R}{1+R} \sigma_{\theta} \\
-\frac{R}{1+R} \sigma_{r}+\sigma_{\theta}
\end{array}\right\}
\end{aligned}
$$

Among several possibilities, the more frequently used Hill non-quadratic yield criterion ${ }^{9}$ for planar isotropic materials with normal anisotropy under plane stress conditions is

$$
\Phi=\frac{1}{2(1+R)}\left[\left|\sigma_{r}+\sigma_{\theta}\right|^{a}+(1+2 R)\left|\sigma_{r}-\sigma_{\theta}\right|^{a}\right]=\bar{\sigma}^{a}
$$

where $\bar{\sigma}$ represents the effective stress defined previously and $R$ is the plastic anisotropy parameter. The exponent $a$ could be an arbitrary positive number larger than or equal to one without losing convexity. ${ }^{23}$ The Hill quadratic yield criterion is recovered from equation (20) when $a=2$. The vector $\mathbf{f}$ corresponding to the Hill non-quadratic yield criterion can be obtained via equation (18) as

$$
\mathbf{f}=\frac{1}{\bar{\sigma}^{a-1}} \frac{1}{2(1+R)}\left\{\begin{array}{l}
\left|\sigma_{r}+\sigma_{\theta}\right|^{a-1} \operatorname{sgn}\left(\sigma_{r}+\sigma_{\theta}\right)+(1+2 R)\left|\sigma_{r}-\sigma_{\theta}\right|^{a-1} \operatorname{sgn}\left(\sigma_{r}-\sigma_{\theta}\right) \\
\left|\sigma_{r}+\sigma_{\theta}\right|^{a-1} \operatorname{sgn}\left(\sigma_{r}+\sigma_{\theta}\right)-(1+2 R)\left|\sigma_{r}-\sigma_{\theta}\right|^{a-1} \operatorname{sgn}\left(\sigma_{r}-\sigma_{\theta}\right)
\end{array}\right\}
$$

where $\operatorname{sgn}(\cdot)$ represents the sign of $(\cdot)$.

The six-component yield criterion ${ }^{11}$ under plane stress conditions without shear stresses becomes

$$
\Phi=\frac{1}{2}\left(|p|^{a}+|q|^{a}+|p+q|^{a}\right)=\bar{\sigma}^{a}
$$

with

$$
\begin{aligned}
& p=\xi_{1}-\xi_{2} \\
& q=\xi_{2}-\xi_{3}
\end{aligned}
$$

Here $\bar{\sigma}$ represents the effective stress defined previously, and $\xi_{1}, \xi_{2}$ and $\xi_{3}$ are the roots of the following equation:

$$
\xi^{3}-3 I_{2} \xi-2 I_{3}=0
$$

with

$$
\begin{gathered}
I_{2}=\frac{\left[c_{3}\left(x_{1}-x_{2}\right)-c_{2}\left(x_{3}-x_{1}\right)\right]^{2}}{54}+\frac{\left[c_{1}\left(x_{2}-x_{3}\right)-c_{3}\left(x_{1}-x_{2}\right)\right]^{2}}{54} \\
+\frac{\left[c_{2}\left(x_{3}-x_{1}\right)-c_{1}\left(x_{2}-x_{3}\right)\right]^{2}}{54} \\
I_{3}=\frac{\left[c_{3}\left(x_{1}-x_{2}\right)-c_{2}\left(x_{3}-x_{1}\right)\right]\left[c_{1}\left(x_{2}-x_{3}\right)-c_{3}\left(x_{1}-x_{2}\right)\right]\left[c_{2}\left(x_{3}-x_{1}\right)-c_{1}\left(x_{2}-x_{3}\right)\right]}{54}
\end{gathered}
$$


where $x_{1}=\left(2 \sigma_{r}-\sigma_{\theta}\right) / 3, x_{2}=\left(2 \sigma_{\theta}-\sigma_{r}\right) / 3$ and $x_{3}=\left(-\sigma_{r}-\sigma_{\theta}\right) / 3$. The exponent $a$ in equation (22) is an arbitrary number larger than or equal to 1 without losing convexity. ${ }^{11}$ It should be noted that the Tresca yield criterion is recovered when $a=1$ or $\infty$ and the von Mises yield criterion is recovered when $a=2$ or 4 for all anisotropic constants $c_{1}, c_{2}$ and $c_{3}$ being equal to one. The details to determine anisotropic constants are given in Appendix. Note $c_{1}=c_{2}$ due to planar isotropy and normal anisotropy. Solving equation (25) with the aids of equations (26) and (27) gives

$$
\begin{aligned}
& \xi_{1}=2 \sqrt{I_{2}} \cos \left(\frac{\theta}{3}\right) \\
& \xi_{2}=2 \sqrt{I_{2}} \cos \left(\frac{\theta-2 \pi}{3}\right) \\
& \xi_{3}=2 \sqrt{I_{2}} \cos \left(\frac{\theta+2 \pi}{3}\right)
\end{aligned}
$$

where $\theta\left(=\cos ^{-1}\left(I_{3} / I_{2}^{3 / 2}\right)\right)$ ranges between 0 and $\pi$.

The plastic strain rates are defined in equation (14) and the vector $\mathbf{f}$ can be obtained via equation (18). Note that $\left(\partial \Phi / \partial \sigma_{r}\right)$ and $\left(\partial \Phi / \partial \sigma_{\theta}\right)$ can be obtained by the chain rule using equations (22) $-(30)$ as

$$
\begin{aligned}
& \frac{\partial \Phi}{\partial \sigma_{r}}=\left(\frac{\partial \Phi}{\partial p} \frac{\partial p}{\partial \xi_{k}}+\frac{\partial \Phi}{\partial q} \frac{\partial q}{\partial \xi_{k}}\right)\left(\frac{\partial \xi_{k}}{\partial I_{2}} \frac{\partial I_{2}}{\partial \sigma_{r}}+\frac{\partial \xi_{k}}{\partial I_{3}} \frac{\partial I_{3}}{\partial \sigma_{r}}\right) \\
& \frac{\partial \Phi}{\partial \sigma_{\theta}}=\left(\frac{\partial \Phi}{\partial p} \frac{\partial p}{\partial \xi_{k}}+\frac{\partial \Phi}{\partial q} \frac{\partial q}{\partial \xi_{k}}\right)\left(\frac{\partial \xi_{k}}{\partial I_{2}} \frac{\partial I_{2}}{\partial \sigma_{\theta}}+\frac{\partial \xi_{k}}{\partial I_{3}} \frac{\partial I_{3}}{\partial \sigma_{\theta}}\right)
\end{aligned}
$$

where the roman subscripts have a range of 1 to 3 . Note that the repeated subscript represents the summation.

As in Peirce et al., ${ }^{22}$ the effective plastic strain rate $\dot{\bar{\varepsilon}}$ p can be derived via Taylor's series expansion when rate sensitivity is accounted for

$$
\dot{\bar{\varepsilon}}^{\mathrm{p}}=\frac{\dot{\bar{\varepsilon}}_{t}^{\mathrm{p}}}{1+\zeta}+\frac{1}{h} \mathbf{F} \cdot \dot{\boldsymbol{\varepsilon}} \frac{\zeta}{1+\zeta}
$$

Here $\dot{\bar{\varepsilon}}_{t}^{\mathrm{p}}$ represents the effective plastic strain rate at time $t, \mathbf{F}$ is defined by the inner product of the elastic modulus tensor $\mathbf{L}$ in equation (13) and the vector $\mathbf{f}$ in equation (15) as

$$
\mathbf{F}=\mathbf{L} \cdot \mathbf{f}=\mathbf{f} \cdot \mathbf{L}
$$

$\dot{\boldsymbol{\varepsilon}}$ is the vector form of the total strain rates

$$
\dot{\boldsymbol{\varepsilon}}=\left\{\begin{array}{c}
\dot{\varepsilon}_{r} \\
\dot{\varepsilon}_{\theta}
\end{array}\right\}
$$

and scalars $h$ and $\zeta$ are defined as

$$
h=\mathbf{f} \cdot \mathbf{L} \cdot \mathbf{f}-\left(\frac{\partial \dot{\bar{\varepsilon}}_{t}^{\mathrm{p}}}{\partial \bar{\varepsilon}^{\mathrm{p}}}\right)\left(\frac{\partial \dot{\bar{\varepsilon}}_{t}^{\mathrm{p}}}{\partial \bar{\sigma}}\right)^{-1}
$$




$$
\zeta=(\Theta \Delta t) h \frac{\partial \dot{\bar{\varepsilon}}_{t}^{\mathrm{p}}}{\partial \bar{\sigma}}
$$

where a parameter $\Theta$ ranges from 0 to 1 . Note that $\Theta=0$ gives the Euler intergration.

The following viscoplastic stress-strain relation is adopted in the present study

$$
\dot{\bar{\varepsilon}}_{t}^{\mathrm{p}}=\dot{\varepsilon}_{0}\left(\frac{\bar{\sigma}}{g}\right)^{1 / m}
$$

Here $m$ is the rate sensitivity and $g$, characterizing the strain hardening, is given by a power-law relation as

$$
g=\sigma_{0}\left(\frac{\bar{\varepsilon}^{\mathrm{p}}}{\varepsilon_{0}}+1\right)^{n}
$$

where $n$ is a strain hardening exponent, $\sigma_{0}$ and $\varepsilon_{0}$ are, respectively, the reference stress and the reference strain related by Young's modulus $E$ as $\sigma_{0}=E \varepsilon_{0}$. Substituting equations (12), (14) and (33) into equations (10) and (11) gives the constitutive response for the stress rates and the strain rates as

$$
\dot{\boldsymbol{\sigma}}=\mathbf{L}^{\mathrm{tan}} \cdot \dot{\boldsymbol{\varepsilon}}-\frac{\dot{\bar{\varepsilon}}_{t}^{\mathrm{p}}}{1+\zeta} \mathbf{F}
$$

where $\dot{\boldsymbol{\sigma}}$ is the vector form of the stress rates

$$
\dot{\boldsymbol{\sigma}}=\left\{\begin{array}{c}
\dot{\sigma}_{r} \\
\dot{\sigma}_{\theta}
\end{array}\right\}
$$

and the tangent modulus matrix $\mathbf{L}^{\text {tan }}$ is defined as

$$
\mathbf{L}^{\tan }=\mathbf{L}-\left(\frac{\zeta}{1+\zeta}\right) \frac{1}{h} \mathbf{F F}
$$

The details of the derivation can be found in Peirce et al. $^{22}$

\subsection{Finite element formulation}

The virtual work principle in terms of the physical components of the stress measure and the strain rate measure for the axisymmetric and plane strain shell elements can be derived as (for example, see the works of Budiansky, ${ }^{24}$ Hutchinson $^{25}$ and Wang and Tang $^{3}$ )

$$
\int_{A_{0}} \int_{-h_{0} / 2}^{h_{0} / 2}\left(\sigma_{r} \delta \dot{\varepsilon}_{r}+\sigma_{\theta} \delta \dot{\varepsilon}_{\theta}\right) \mathrm{d} \eta \mathrm{d} A_{0}=\int_{A_{0}}\left(N_{1} \delta \dot{u}+N_{3} \delta \dot{w}\right) \mathrm{d} A_{0}
$$

where $h_{0}$ is the initial sheet thickness, $\mathrm{d} A_{0}$ is the differential area in the undeformed configuration, $N_{1}$ and $N_{3}$ are the components of the current surface load in the $\rho$ and $\gamma$ direction, respectively. The variational equation can be derived via equation (43) by following the same procedure in Budiansky. ${ }^{24}$ Substituting the constitutive equation (40) and the kinematics relations described previously into the variational equation gives the resulting stiffness equation in the matrix form as

$$
\mathbf{K} \dot{\mathbf{U}}=\dot{\mathbf{Q}}+\dot{\mathbf{Q}}^{(\mathrm{vp})}
$$

Int. J. Numer. Meth. Engng., 41, 559-584 (1998)

(C) 1998 John Wiley \& Sons, Ltd. 
where $\mathbf{K}$ is the global stiffness matrix, $\dot{\mathbf{U}}$ is the nodal velocity vector, $\dot{\mathbf{Q}}$ is the nodal force rate vector, and $\dot{\mathbf{Q}}^{(\mathrm{vp})}$ is the viscoplastic force rate vector. Note that Hermitian cubic polynomials are used as the interpolation functions for $u$ and $w$ whereas three-point Simpson's rule is used for the numerical integration over the element domain $\mathrm{d} A_{0}$. The nodal velocity vector $\dot{\mathbf{U}}$ of the $i$ th node is expressed as

$$
\dot{\mathbf{U}}^{(i) \mathrm{T}}=\left(\dot{u}^{(i)}, \frac{\partial \dot{u}^{(i)}}{\partial x}, \dot{w}^{(i)}, \frac{\partial \dot{w}^{(i)}}{\partial x}\right)
$$

where the superscript $\mathrm{T}$ represents the 'transpose'. The contribution from the $I$ th element to the global stiffness matrix $\mathbf{K}$, without containing the $\dot{z}$ term in equation (7), can be expressed as

$$
\mathbf{K}^{(E(I))}=\int_{A_{0}} \int_{-h_{0} / 2}^{h_{0} / 2} \mathbf{N}^{\mathrm{T}}\left(\mathbf{H}^{\mathrm{T}} \mathbf{L}^{\tan } \mathbf{H}+\sigma_{r} \mathbf{K}^{(1)}+\sigma_{\theta} \mathbf{K}^{(2)}\right) \mathbf{N} \mathrm{d} \eta \mathrm{d} A_{0}
$$

where the superscript $E(I)$ represents the corresponding quantity contributed from the $I$ th element. $\mathbf{N}$ is a $6 \times 8$ element interpolation matrix defined by

$$
\begin{aligned}
\dot{\mathbf{u}} & =\mathbf{N} \dot{\mathbf{u}}^{(E(I)) \mathrm{T}} \\
\dot{\mathbf{u}}^{\mathrm{T}} & =\left(\dot{u}, \dot{w}, \frac{\partial \dot{u}}{\partial x}, \frac{\partial \dot{w}}{\partial x}, \frac{\partial^{2} \dot{u}}{\partial x^{2}}, \frac{\partial^{2} \dot{w}}{\partial x^{2}}\right) \\
\dot{\mathbf{u}}^{(E(I)) \mathrm{T}} & =\left(\dot{\mathbf{u}}^{(I) \mathrm{T}}, \dot{\mathbf{u}}^{(I+1) \mathrm{T}}\right)
\end{aligned}
$$

and $\mathbf{H}$ is a $2 \times 6$ matrix defined by

$$
\mathbf{H}=\left(\mathbf{g}^{\mathrm{T}}, \mathbf{h}^{\mathrm{T}}, \mathbf{p}^{\mathrm{T}}, \mathbf{q}^{\mathrm{T}}\right)^{\mathrm{T}}
$$

Note that $\mathbf{g}^{\mathrm{T}}, \mathbf{h}^{\mathrm{T}}, \mathbf{p}^{\mathrm{T}}$ and $\mathbf{q}^{\mathrm{T}}$ in equation (50), $\mathbf{K}^{(1)}$ and $\mathbf{K}^{(2)}$ in equation (46) as well as a $8 \times 1$ force rate vector contributed from the $I$ th element $\mathbf{Q}^{(E(I))}$ including $\dot{z}$ term in equation (7) are all listed in Wang and Tang. ${ }^{3}$ From the variational equation, a $8 \times 1$ viscoplastic force rate vector contributed from the $I$ th element $\dot{\mathbf{Q}}^{(\mathrm{vp})(E(I))}$ is obtained as

$$
\dot{\mathbf{Q}}^{(\mathrm{vp})(E(I))}=\int_{A_{0}} \int_{-h_{0} / 2}^{h_{0} / 2} \mathbf{N}^{\mathrm{T}} \mathbf{H}^{\mathrm{T}}\left(\frac{\dot{\bar{\varepsilon}}_{t}^{\mathrm{p}}}{1+\zeta}\right) \mathbf{F} \mathrm{d} \eta \mathrm{d} A_{0}
$$

The contact constraint and equilibrium check of Tang ${ }^{2}$ are adopted to simulate the contact process. Note that the external bending moments at any contact nodes are set to equal zero.

\section{APPLICATIONS}

\subsection{Hemispherical punch stretching}

A hemispherical punch stretching operation is schematically shown in Figure 2. A piece of sheet metal is considered to be pressed by a hemispherical punch through a circular arc-flanged die. The geometric parameters of the punch stretching operation are $r_{\mathrm{p}}$ (the punch profile radius) $=50.8 \mathrm{~mm}$, $r_{\mathrm{d}}$ (the die profile radius) $=6.35 \mathrm{~mm}, r_{0}$ (the punch opening radius) $=52.83 \mathrm{~mm}$, and $h_{0}$ (the sheet thickness $)=1.2 \mathrm{~mm}$. The restraining force due to the binder is simulated by a stiff spring to control 


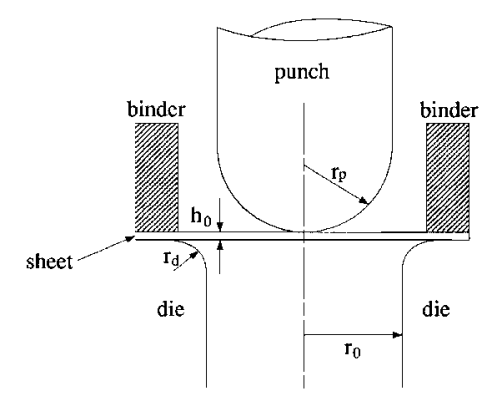

Figure 2. A hemispherical punch stretching operation

the flow of metal into the die. The equivalent spring constant, used to substitute the frictional force due to the binder pressure, is set at $1 \mathrm{GN} / \mathrm{mm}$. Both the punch and die are assumed to be rigid.

Note that the effect of the friction on the strain distribution is significant for metal forming operations (for example, see the work of Wang and Wenner ${ }^{21}$ ). However, we only concentrate on the effect of the yield criteria on the strain distribution in this study. Therefore, the friction coefficient $\mu$ in Coulomb's friction law for contact surfaces is kept to be constant $(\mu=0 \cdot 17)$. We take the material parameters $E=206 \mathrm{GPa}, \sigma_{0}=103.3 \mathrm{MP}, v=0.3, n=0.21, m=0.012$ and $R=1.8$ to represent a typical low-carbon steel. We take $E=70 \mathrm{GPa}, \sigma_{0}=50.0 \mathrm{MPa}, v=0.34$, $n=0.22, m=0.005$ and $R=0.8$ to represent an aluminum alloy. ${ }^{26,27}$ The exponent $a$ of the Hill non-quadratic yield criterion is selected to be 2.3 for $R=1.8$ for the low-carbon steel and $a=1.6$ for $R=0.8$ for the aluminum as in Liao et al. ${ }^{28}$ As in Logan and Hosford, ${ }^{29}$ the exponent $a$ of the six-component yield criterion is selected to be 6.0 and 8.0 for the low-carbon steel (b.c.c. metal) and the aluminum (f.c.c. metal), respectively. Three anisotropic constants for the six-component yield criterion can be determined by using the yield stress under the in-plane uniaxial tensile loading, the plastic anisotropy parameter $R$ and the condition of $c_{1}=c_{2}$. Then $\sigma_{0}=103.3 \mathrm{MPa}$ and $R=1.8$ give $c_{1}=c_{2}=0.883$ and $c_{3}=1.110$ for the low-carbon steel whereas $\sigma_{0}=50.0 \mathrm{MPa}$ and $R=0.8$ give $c_{1}=c_{2}=1.033$ and $c_{3}=0.966$ for the aluminum.

For the convenience of presentation, the Hill quadratic yield criterion, the Hill non-quadratic yield criterion, and the six-component yield criterion are designated as criterion HQ, HNQ, and 6C, respectively. Figure 3 shows the yield surfaces based on criterion $\mathrm{HQ}, \mathrm{HNQ}$, and 6C for the low-carbon steel $(R=1.8)$ in the normalized principal stress plane. In the figure, the in-plane principal stresses are denoted as $\sigma_{1}$ and $\sigma_{2}$ and the yield stress under the in-plane uniaxial tensile loading is denoted as $\bar{\sigma}$. Due to planar isotropy, only a quarter of the yield surface is shown. In Figure 3, the yield surface shapes and sizes based on the three yield criteria are quite different near the equal biaxial tensile state. We adopt the ratio of the plane strain yield stress to the equal biaxial yield stress as in Barlat ${ }^{15}$ to characterize the yield surface shape. The ratios based on criterion $\mathrm{HQ}, \mathrm{HNQ}$, and $6 \mathrm{C}$ are obtained as $1 \cdot 103,1 \cdot 164$, and 1.081 , respectively. Note that the low ratio promotes strain localization under in-plane biaxial loading conditions. ${ }^{15}$

Figure 4(a) shows the thickness strain distributions as functions of the initial material position to the centre for the low-carbon steel with $R=1.8$ based on criterion $\mathrm{HQ}, \mathrm{HNQ}$, and 6C at the punch travel distance $d=36 \mathrm{~mm}$. Note that the negative thickness strains are plotted in the figure. The strain distributions and the peak strain positions based on the three criteria are quite different for the given $R=1 \cdot 8$. Our computations show that as $d$ increases, the peak strain 


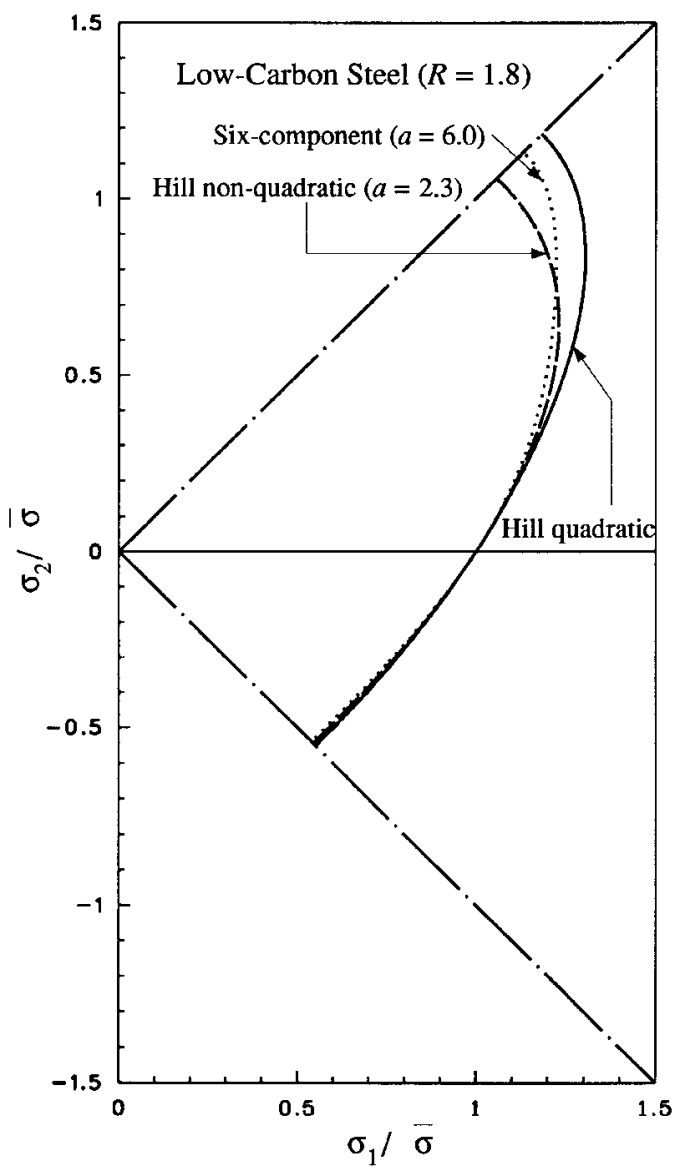

Figure 3. The yield surfaces based on criterion $\mathrm{HQ}, \mathrm{HNQ}$, and $6 \mathrm{C}$ for the low-carbon steel $(R=1.8)$ in the normalized principal stress plane

position moves outward from the central area of the sheet, where near equal biaxial tension occurs. In Figure 4(a), criterion 6C gives a significant peak strain which is clearly related to the flatness of the yield surface between the equal biaxial tension and plane strain states by the low ratio (1.081) of the plane strain yield stress to the equal biaxial yield stress. Figure 4(b) shows the deformed position of the sheet metal based on criterion $6 \mathrm{C}$ at the punch travel distance $d=36 \mathrm{~mm}$. In the figure, an arrow indicates the element having the peak strain.

Figure 5(a) shows the strain path for the bottom surface of the critical element having the peak strain of the low-carbon steel sheet with $R=1.8$ based on criterion $6 \mathrm{C}$. Note that the strains of the bottom surface of the sheet are the most critical measurement of the formability for this type of problem. Significant strain localization is observed in the meridian direction as the strain rate in the circumferential direction approaches to zero. A meridian strain of 1.08 of the bottom surface of the sheet is shown in the figure at the punch travel distance $d=36 \mathrm{~mm}$. It should be noted that we are conducting a parametric study here. The steel sheet may have already failed at the meridian strain of about 50 to 60 per cent under much smaller punch travel distances if a finite imperfection 

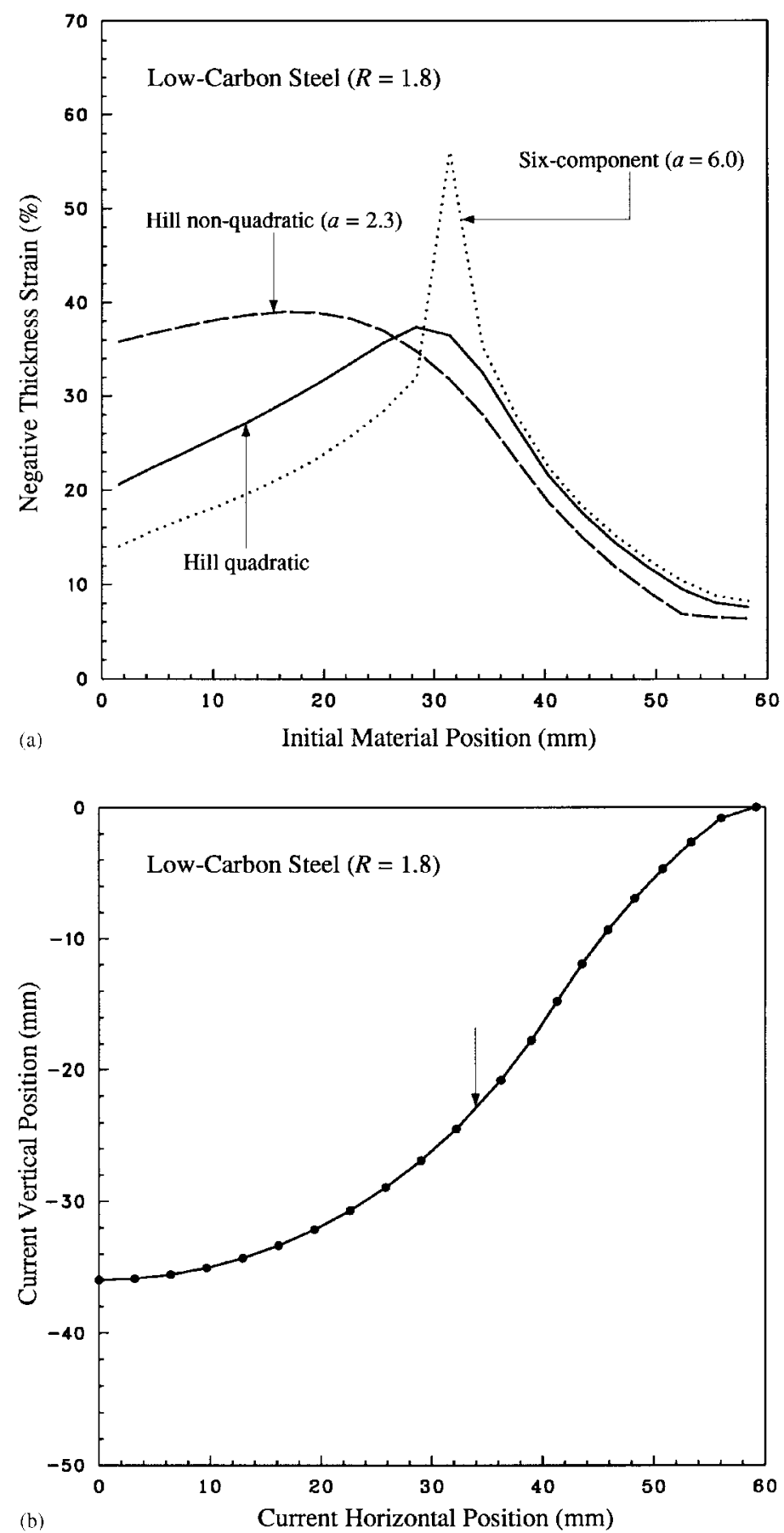

Figure 4. (a) Comparison of the thickness strain distributions as functions of the initial material position for the low-carbon steel with $R=1.8$ based on criterion $\mathrm{HQ}, \mathrm{HNQ}$, and $6 \mathrm{C}$ and (b) the deformed position for the low-carbon steel with $R=1.8$ based on criterion $6 \mathrm{C}$ at $d=36 \mathrm{~mm}$ under the hemispherical punch stretching operation 

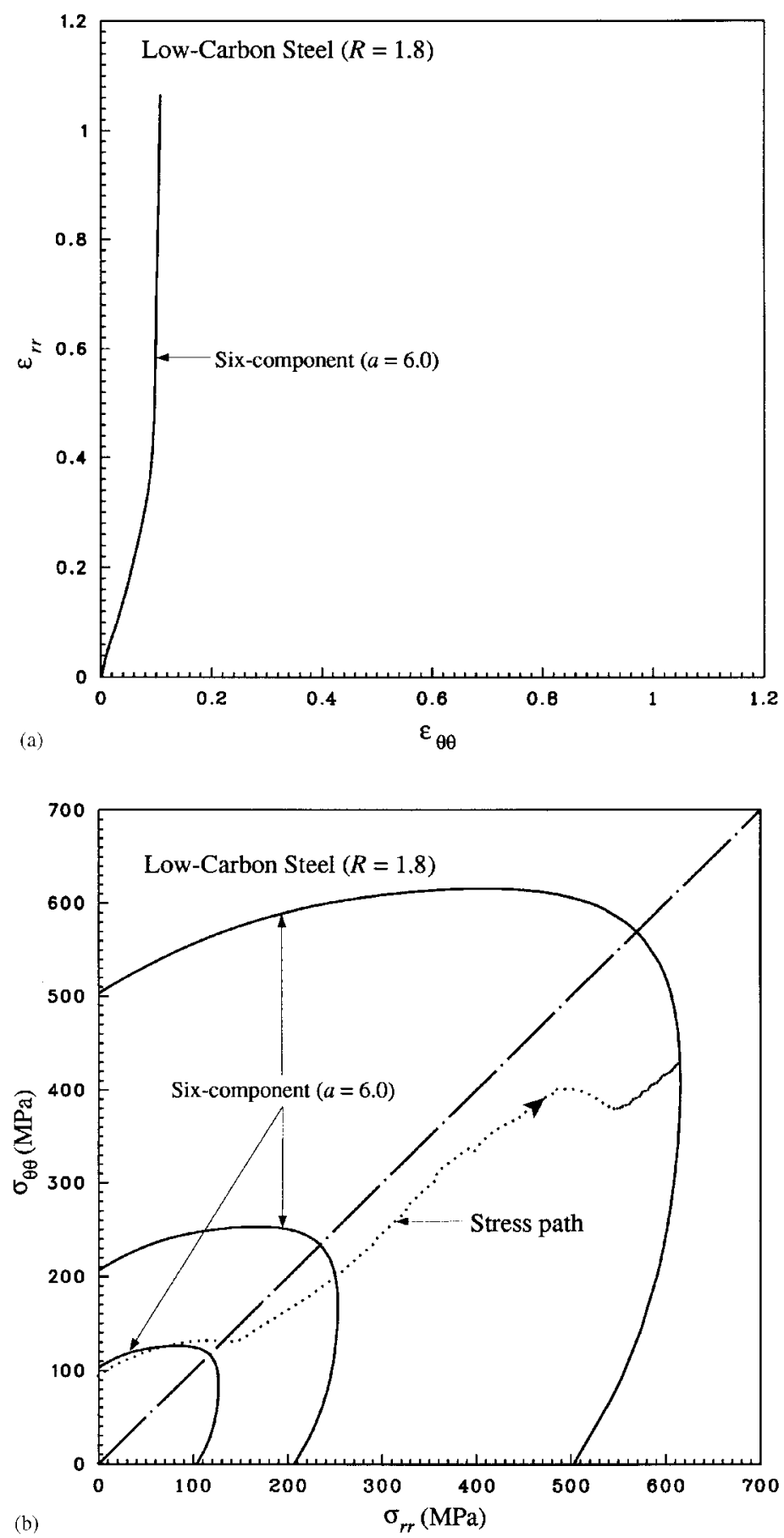

Figure 5. (a) The strain path and (b) the stress path for the bottom surface of the critical element with the peak strain of the low-carbon steel sheet with $R=1.8$ based on criterion $6 \mathrm{C}$ under the hemispherical punch stretching operation 


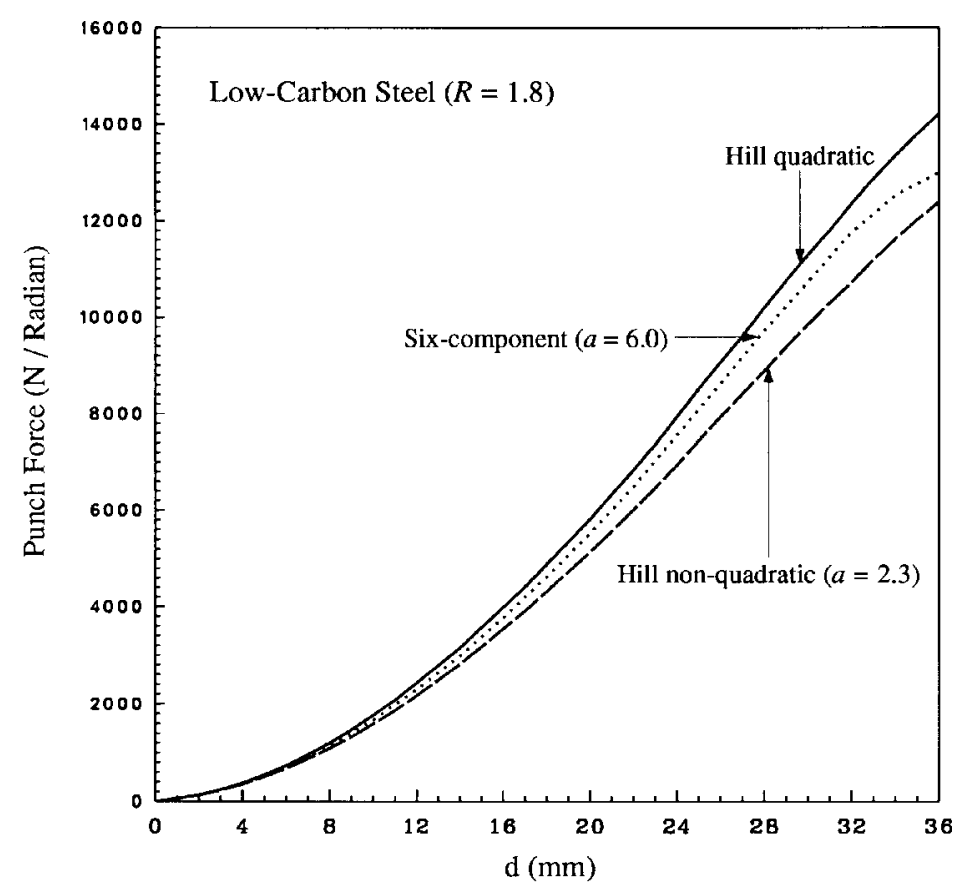

Figure 6. Comparison of the punch forces per unit radian for the low-carbon steel with $R=1.8$ based on criterion HQ, $\mathrm{HNQ}$, and $6 \mathrm{C}$ under the hemispherical punch stretching operation

in the sheet is considered as in the Marciniak-Kuczynski approach. ${ }^{13}$ However, we here examine the effects of yield criteria on the entire strain distribution of the sheet. Figure 5(b) shows the corresponding stress path for the bottom surface of the critical element. Here the yield surfaces based on criterion $6 \mathrm{C}$ are also plotted for reference. Note that the isotropic hardening is assumed in the present study. In Figure 5(b), the loading condition for the critical element is changed from the near equal biaxial tension to the plane strain tension at large punch travel distances. Note that we did not plot the stress path where $\sigma_{r r}$ is negative in the elastic range.

Figure 6 shows the punch forces per unit radian as functions of the punch travel distance $d$ for the low-carbon steel with $R=1.8$ based on criterion $\mathrm{HQ}, \mathrm{HNQ}$, and 6C. For the three yield criteria, the larger punch force at a given punch travel distance is due to the higher yield stresses between the equal biaxial and plane strain states. Therefore criterion HQ gives the largest punch force whereas HNQ gives the smallest punch force at a given punch travel distance. The increasing rate of the punch force based on criterion 6C decreases apparently at $d=36 \mathrm{~mm}$ where significant necking (large thickness strain) occurs as shown in Figure 4(a).

Figure 7 shows the yield surfaces based on criterion $\mathrm{HQ}, \mathrm{HNQ}$, and $6 \mathrm{C}$ for the aluminum $(R=0 \cdot 8)$ in the normalized principal stress plane. The yield surface shapes and sizes based on these three yield criteria are quite different near the equal biaxial tensile and pure shear states. The ratios of the plane strain yield stress to the equal biaxial yield stress based on criterion HQ, $\mathrm{HNQ}$, and $6 \mathrm{C}$ are obtained as $1 \cdot 176,1 \cdot 070$, and $1 \cdot 103$, respectively.

Figure 8(a) shows the thickness strain distributions as functions of the initial material position to the centre for the aluminum with $R=0.8$ based on criterion $\mathrm{HQ}, \mathrm{HNQ}$, and $6 \mathrm{C}$ at $d=32 \mathrm{~mm}$. 


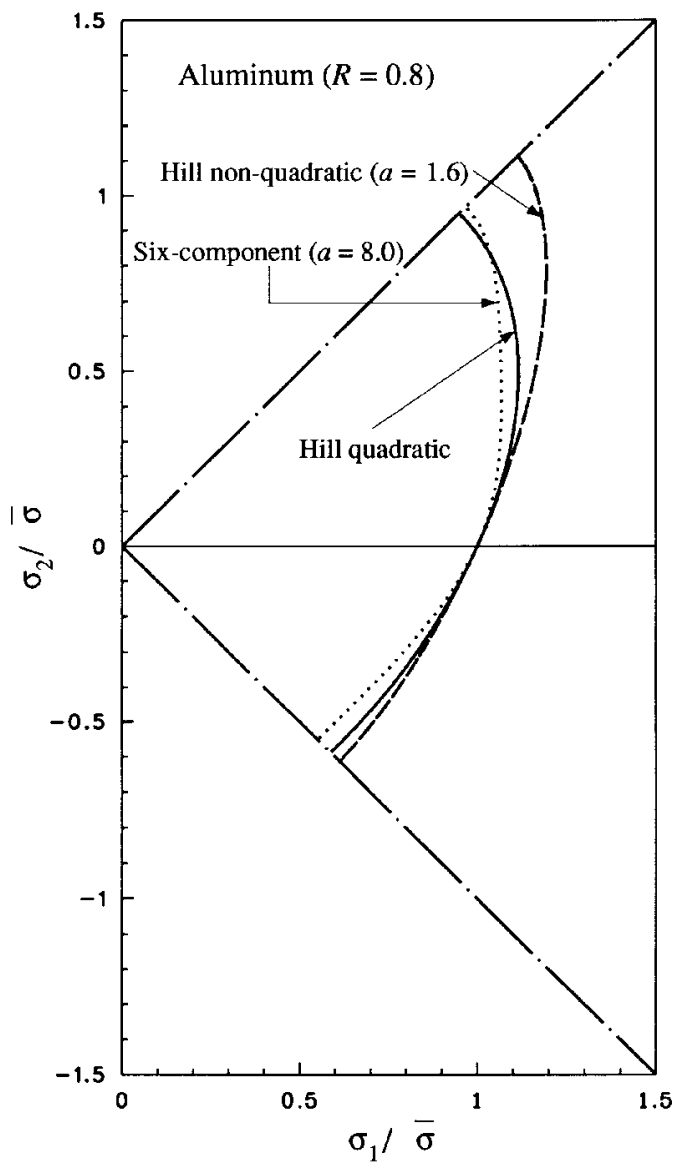

Figure 7. The yield surfaces based on criterion $\mathrm{HQ}, \mathrm{HNQ}$, and $6 \mathrm{C}$ for the aluminum $(R=0 \cdot 8)$ in the normalized principal stress plane

The results of the simulation based on criterion HNQ display a substantial amount of necking. The results of the simulation based on criterion 6C display a smaller amount of necking. This tendency is clearly related to the flatness of the yield surface near the equal biaxial and plane strain states by the low ratio (1.070) of the plane strain yield stress to the equal biaxial yield stress. Figure 8(b) shows the deformed position of the sheet metal based on criterion HNQ at the punch travel distance $d=32 \mathrm{~mm}$. In the figure, an arrow indicates the element having the peak strain.

Figure 9 (a) shows the strain path for the bottom surface of the critical element having the peak strain of the aluminum sheet with $R=0.8$ based on criterion HNQ. Significant strain localization occurs in the meridian direction and the strain rate in the circumferential direction decreases to zero toward the plane strain state. In the figure, a meridian strain of 0.52 of the bottom surface of the sheet is much larger than the corresponding average thickness strain of 0.38 (as shown in Figure $8(\mathrm{a}))$ at $d=32 \mathrm{~mm}$. It should be noted that we are conducting a parametric study. Our goal is to understand the effects of the yield criteria on the entire strain distribution of the sheet. 

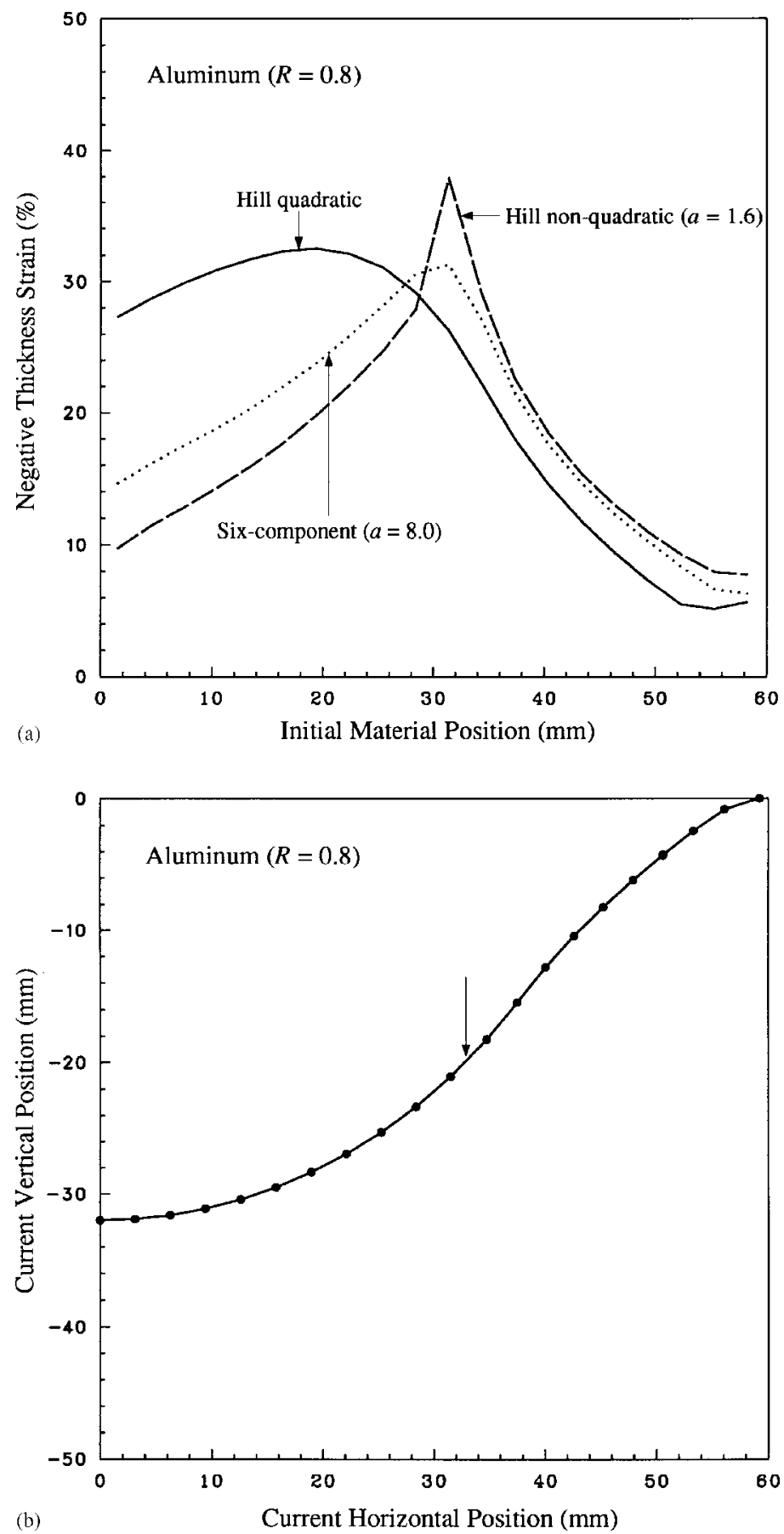

Figure 8. (a) Comparison of the thickness strain distributions as functions of the initial material position for the aluminum with $R=0.8$ based on criterion $\mathrm{HQ}, \mathrm{HNQ}$, and $6 \mathrm{C}$ and (b) the deformed position for the aluminum with $R=0.8$ based on criterion $\mathrm{HNQ}$ at $d=32 \mathrm{~mm}$ under the hemispherical punch stretching operation 

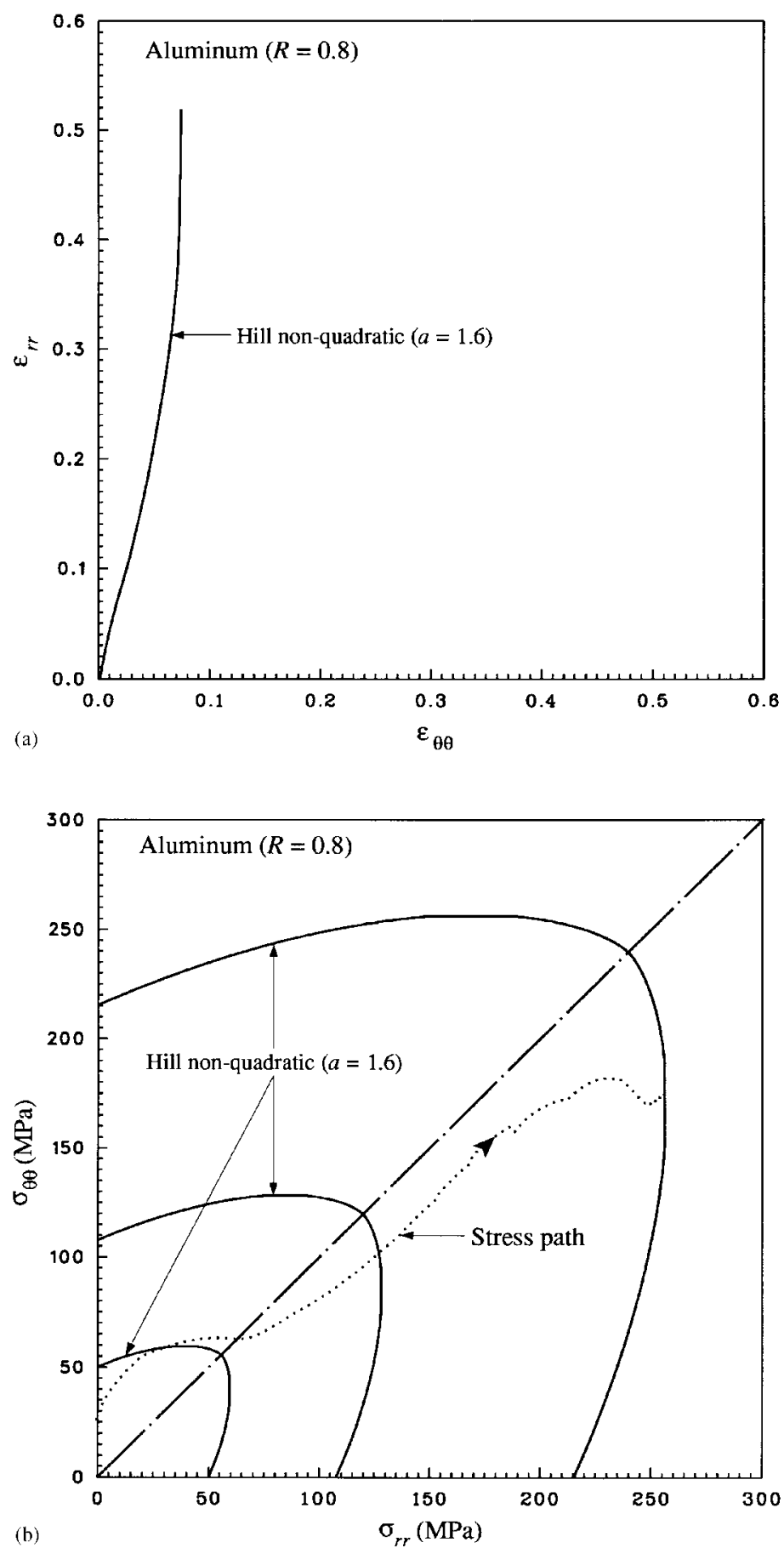

Figure 9. (a) The strain path and (b) the stress path for the bottom surface of the critical element with the peak strain of the aluminum sheet with $R=0.8$ based on criterion HNQ under the hemispherical punch stretching operation 


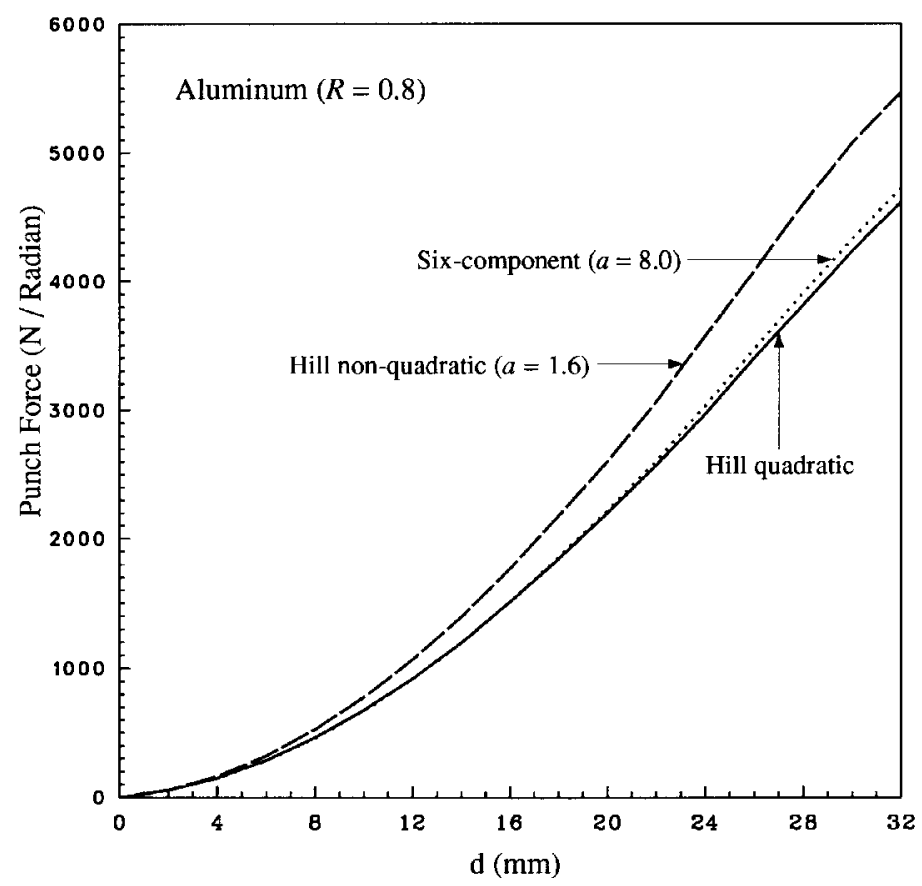

Figure 10. Comparison of the punch forces per unit radian for the aluminum with $R=0.8$ based on criterion HQ, HNQ, and $6 \mathrm{C}$ under the hemispherical punch stretching operation

In reality, the sheet may have already failed at the meridian strain of about 30 to 50 per cent under much smaller punch travel distances if we consider a finite amount of imperfection in the sheet as in the Marciniak-Kuczynski approach. ${ }^{13}$ Figure $9(\mathrm{~b})$ shows the corresponding stress path for the bottom surface of the critical element. Here the yield surfaces based on criterion HNQ are also plotted for reference. The loading condition for the critical element is changed from the nearly biaxial tension to the plane strain tension at large punch travel distances as shown in Figure 9(b). Note that we did not plot the elastic stress state where $\sigma_{r r}$ is negative.

Figure 10 shows the punch forces per unit radian as functions of the punch travel distance $d$ for the aluminum with $R=0.8$ based on criterion $\mathrm{HQ}, \mathrm{HNQ}$, and 6C. As for the case where $R>1$, the punch force is related to the yield stresses between the equal biaxial and plane strain loading conditions. Therefore, criterion HNQ gives the highest punch force whereas criterion HQ and $6 \mathrm{C}$ give virtually the same lower punch forces.

\subsection{Plane strain drawing}

A plane strain drawing operation is shown in Figure 11. The geometric parameters of the plane strain drawing operation are $r_{\mathrm{p}}$ (the punch profile radius) $=5.5 \mathrm{~mm}, r_{\mathrm{d}}$ (the die profile radius $)=10.5 \mathrm{~mm}, \quad w$ (the punch size $)=10.5 \mathrm{~mm}$, and $h_{0}$ (the sheet thickness) $=1.0 \mathrm{~mm}$. The restraining force due to the binder pressure is simulated by a soft elastic-plastic spring. The limit force of the spring is set at a small value of $250 \mathrm{~N} / \mathrm{mm}$ to simulate the drawing operation. Both the punch and die are assumed to be rigid. The friction coefficient $\mu$ in Coulomb's friction 


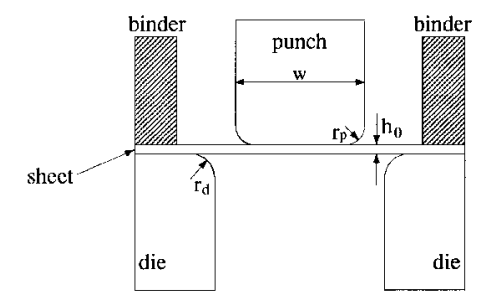

Figure 11. A plane strain drawing operation

law for contact surfaces is assumed to be 0.02 under well-lubricated conditions. The material parameters, the exponent $a$ of the Hill non-quadratic and the six-component yield criteria, and the three anisotropic constants $c_{1}, c_{2}$ and $c_{3}$ of the six-component yield criterion for both the low-carbon steel and the aluminum are the same as those adopted for the hemispherical punch stretching operation.

Figure 12(a) shows the thickness strain distributions as functions of the initial material position to the centreline for the low-carbon steel with $R=1.8$ based on criterion HQ, HNQ, and 6C at the punch travel distance $d=20 \mathrm{~mm}$. There are three peaks near the current positions of the punch and the die corners due to the complex loading paths, such as bending, unbending, and stretching operations. The positions of the three peaks based on criterion $6 \mathrm{C}$ are denoted as $\mathrm{A}, \mathrm{B}$, and $\mathrm{C}$. The results of the simulations based on the three criteria have the same peak positions and trends of the strain distribution. The strain distributions based on criterion HNQ and $6 \mathrm{C}$ are nearly coincident as shown in Figure 12(a) because the plane strain yield stresses are almost the same. Note that the plane strain state is close to the location where the outward normal to the yield surface is parallel to the $\sigma_{1}$ axis when the plastic strain is much larger than the elastic strain. The effect of the yield surface shape for the plane strain drawing operation is not as significant as that for the hemispherical punch stretching operation since only one stress parameter (the plane strain yield stress) is involved. Figure 12(b) shows the deformed position of the sheet metal based on criterion $6 \mathrm{C}$ at the punch travel distance $d=20 \mathrm{~mm}$. In Figure 12(b), $\mathrm{A}, \mathrm{B}$, and $\mathrm{C}$ indicate the corresponding elements having the peak thickness strains as shown in Figure 12(a).

Figure 13(a) shows the strain history for the bottom surface of the critical element having the largest peak strain of the low-carbon steel sheet with $R=1.8$ based on criterion $6 \mathrm{C}$. The selected critical element is located near the punch corner (location A in Figure 12(b)). A slight decrease of the strain due to elastic unloading can be observed at large punch travel distances. Note that the meridian strain of 0.176 on the bottom surface of the sheet is much larger than the average thickness strain $(=0.074$ as shown in Figure 12(a)) at $d=20 \mathrm{~mm}$. This indicates the importance of the bending in the drawing operation. Figure 13(b) shows the corresponding stress path for the bottom surface of the critical element. Here the yield surfaces based on criterion $6 \mathrm{C}$ are also included for reference. As shown in the figure, the bottom surface of the critical element is under nearly proportional loading conditions before elastic unloading. Before and after the elastic unloading, the strains are nearly the same as shown in Figure 13(a) since the elastic strain due to unloading is quite small when compared to the plastic strain. However, the stress states are quite different before and after the elastic unloading as shown in Figure 13(b). This example indicates that accurate constitutive modelling and numerical scheme are needed to predict correctly the stress state in complex elastic-plastic finite element analyses. 

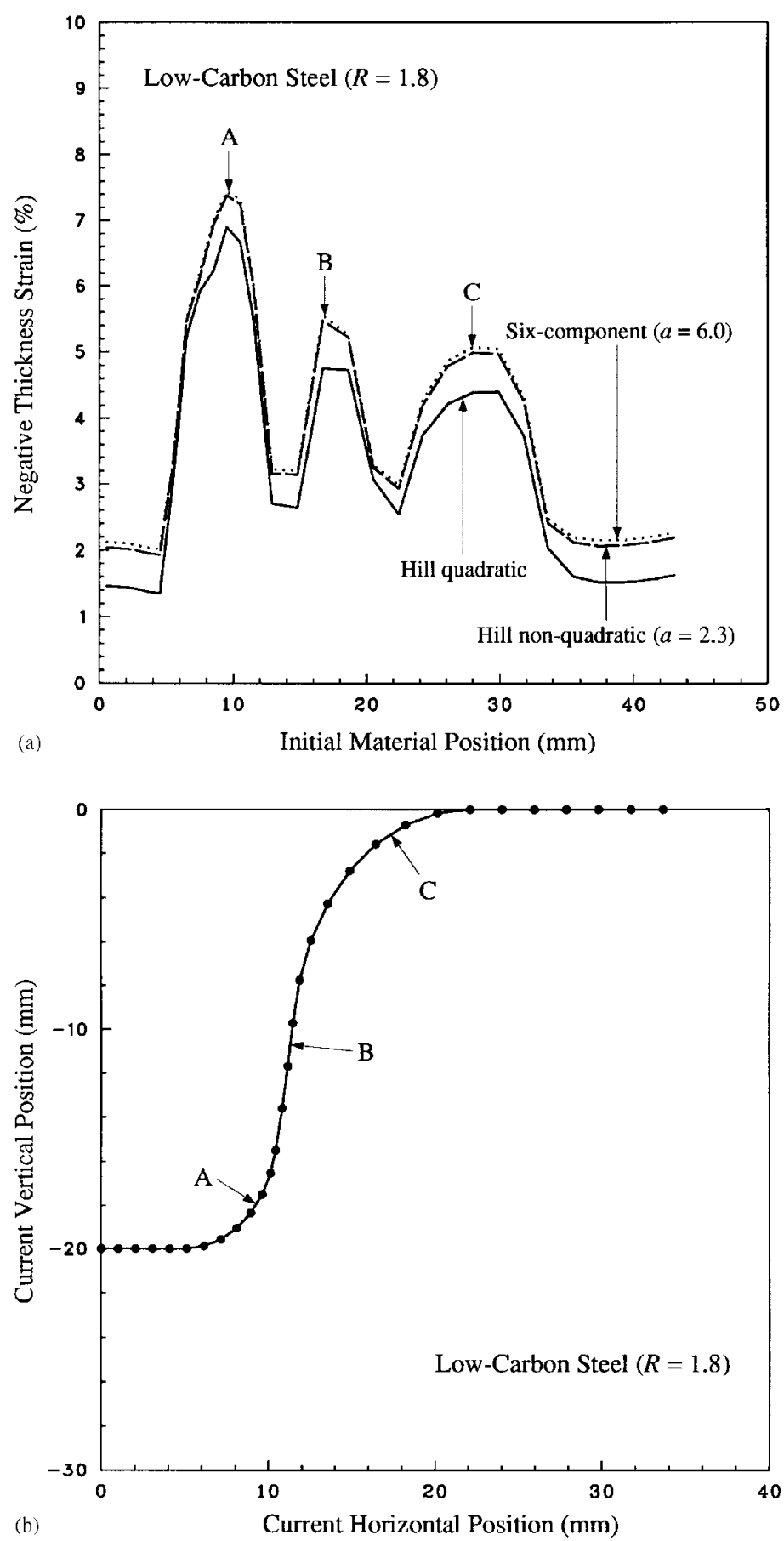

Figure 12. (a) Comparison of the thickness strain distributions as functions of the initial material position for the low-carbon steel with $R=1.8$ based on criterion $\mathrm{HQ}, \mathrm{HNQ}$, and $6 \mathrm{C}$ and (b) the deformed position for the low-carbon steel with $R=1.8$ based on criterion $6 \mathrm{C}$ at $d=20 \mathrm{~mm}$ under the plane strain drawing operation 

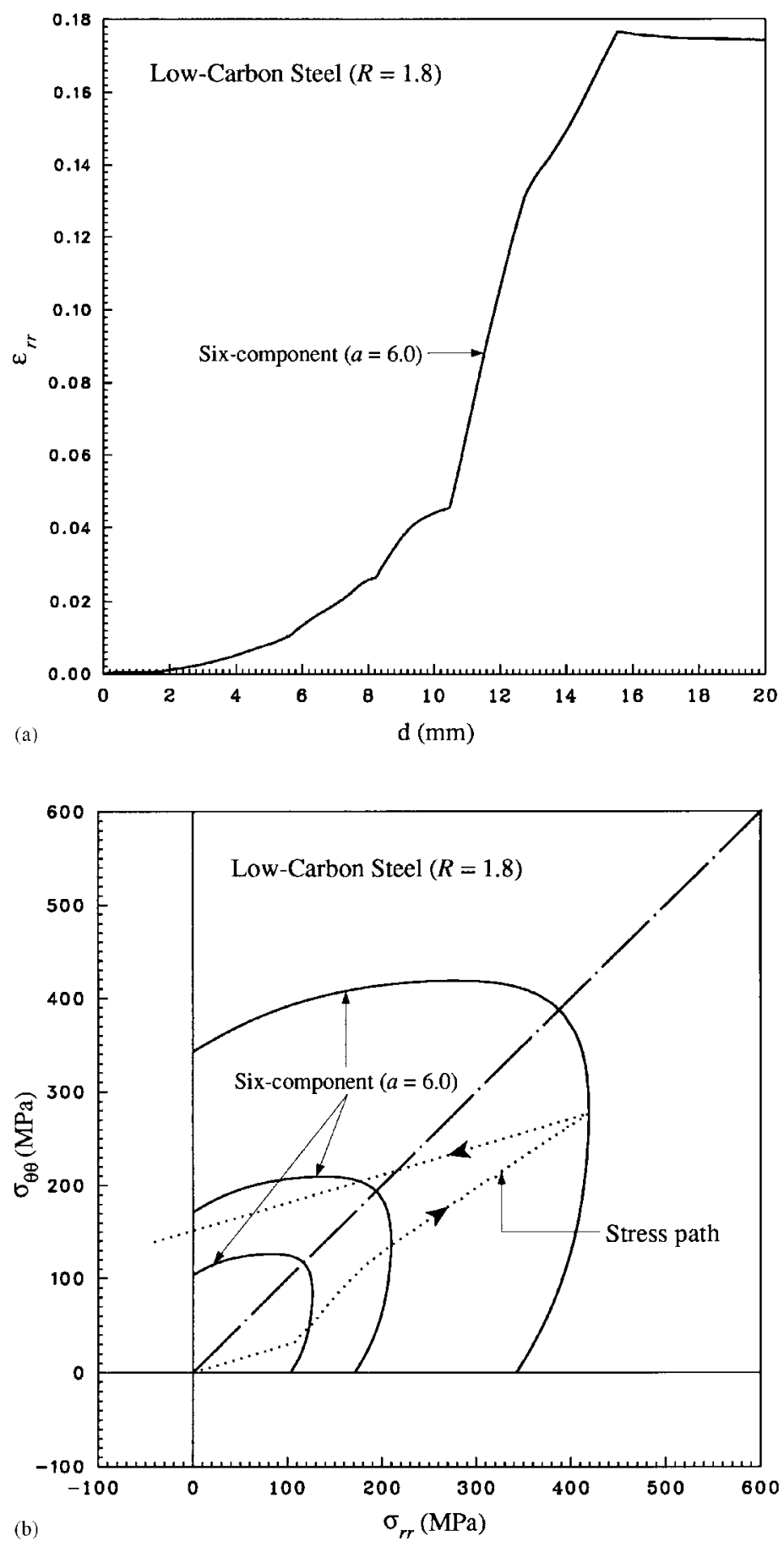

Figure 13. (a) The strain history and (b) the stress path for the bottom surface of the critical element with the largest peak strain of the low-carbon steel sheet with $R=1.8$ based on criterion $6 \mathrm{C}$ under the plane strain drawing operation 


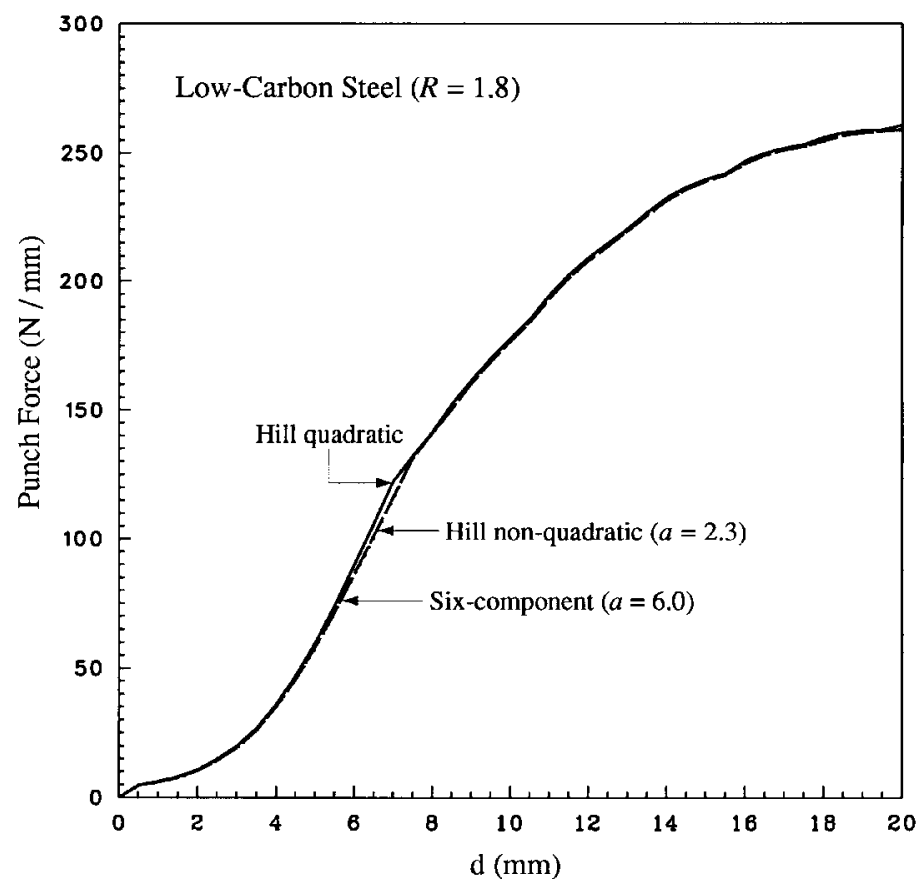

Figure 14. Comparison of the punch forces per unit width for the low-carbon steel with $R=1.8$ based on criterion HQ, $\mathrm{HNQ}$, and $6 \mathrm{C}$ under the plane strain drawing operation

Figure 14 shows the punch forces per unit width as functions of the punch travel distance $d$ for the steel with $R=1.8$ based on criterion $\mathrm{HQ}, \mathrm{HNQ}$, and 6C. The differences of the punch forces based on these three criteria are quite small whereas criterion HNQ and 6C give almost the same punch forces as shown in the figure.

Figure 15(a) shows the thickness strain distributions as functions of the initial material position to the centreline for the aluminum with $R=0.8$ based on criterion $\mathrm{HQ}, \mathrm{HNQ}$, and $6 \mathrm{C}$ at the punch travel distance $d=10 \mathrm{~mm}$. In the figure, two peaks are displayed. The positions of the two peaks based on criterion $6 \mathrm{C}$ are denoted as $\mathrm{A}$ and $\mathrm{B}$. There are no significant differences of the results based on the three criteria. Figure 15(b) shows the deformed position of the sheet metal based on criterion $6 \mathrm{C}$ at the punch travel distance $d=10 \mathrm{~mm}$. In Figure $15(\mathrm{~b}), \mathrm{A}$ and B indicate the elements having the peak strains as shown in Figure 15(a).

The critical element having the largest peak strain of the aluminum sheet with $R=0.8$ is located near the punch corner (location A in Figure 15(b)). The strain history and stress path for the bottom surface of the critical element are quite similar to those shown in Figures 13(a) and 13(b) but without elastic unloading. Therefore the strain history and stress path are not shown here. Figure 16 shows the punch forces per unit width as functions of the punch travel distance $d$ for the aluminum with $R=0.8$ based on criterion HQ, HNQ, and 6C. As for the steel sheets, the differences of the punch forces based on the three yield criteria at a given $d$ are quite small. 

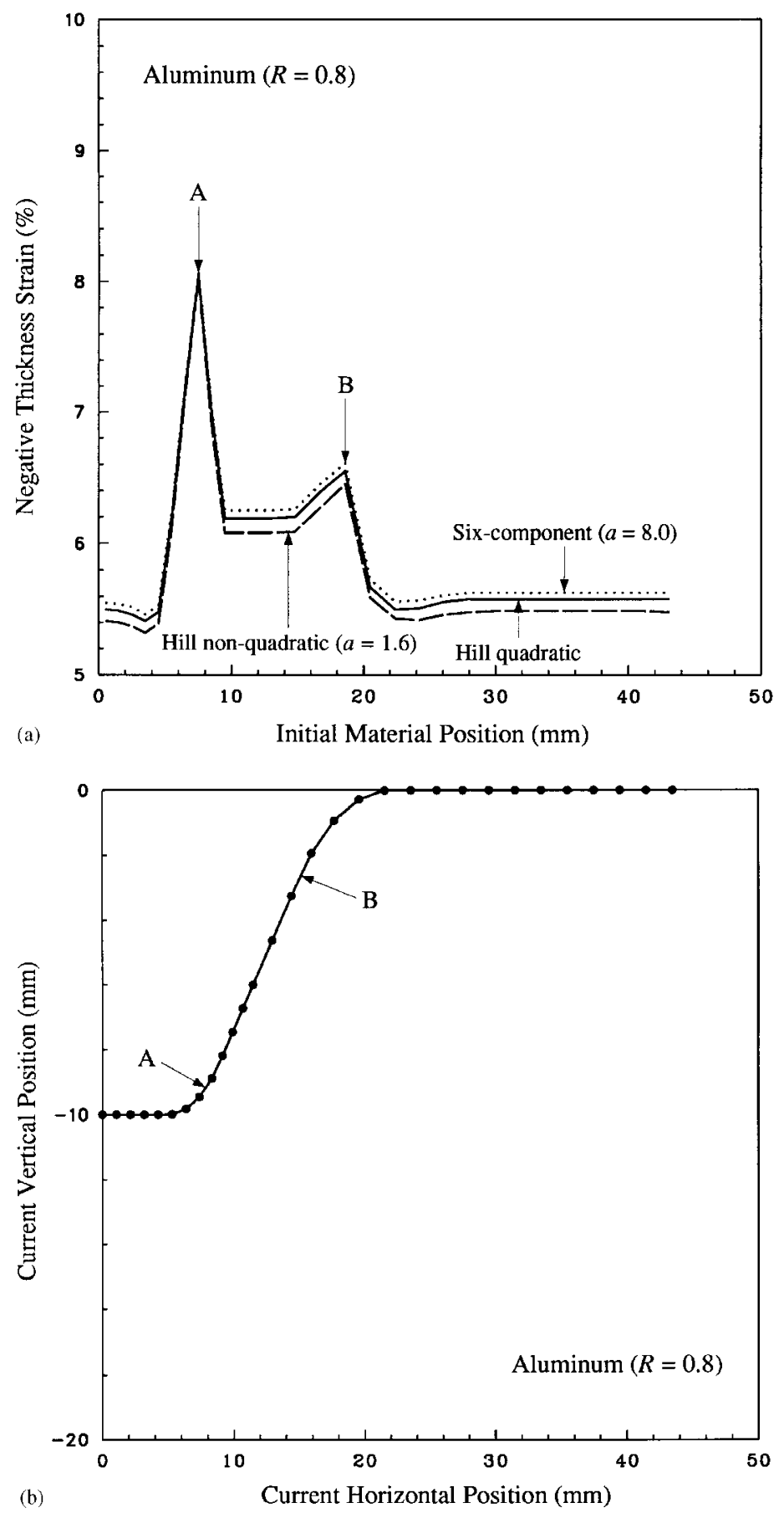

Figure 15. (a) Comparison of the thickness strain distributions as functions of the initial material position for the aluminum with $R=0.8$ based on criterion $\mathrm{HQ}, \mathrm{HNQ}$, and $6 \mathrm{C}$ and (b) the deformed position for the aluminum with $R=0.8$ based on criterion $6 \mathrm{C}$ at $d=10 \mathrm{~mm}$ under the plane strain drawing operation 


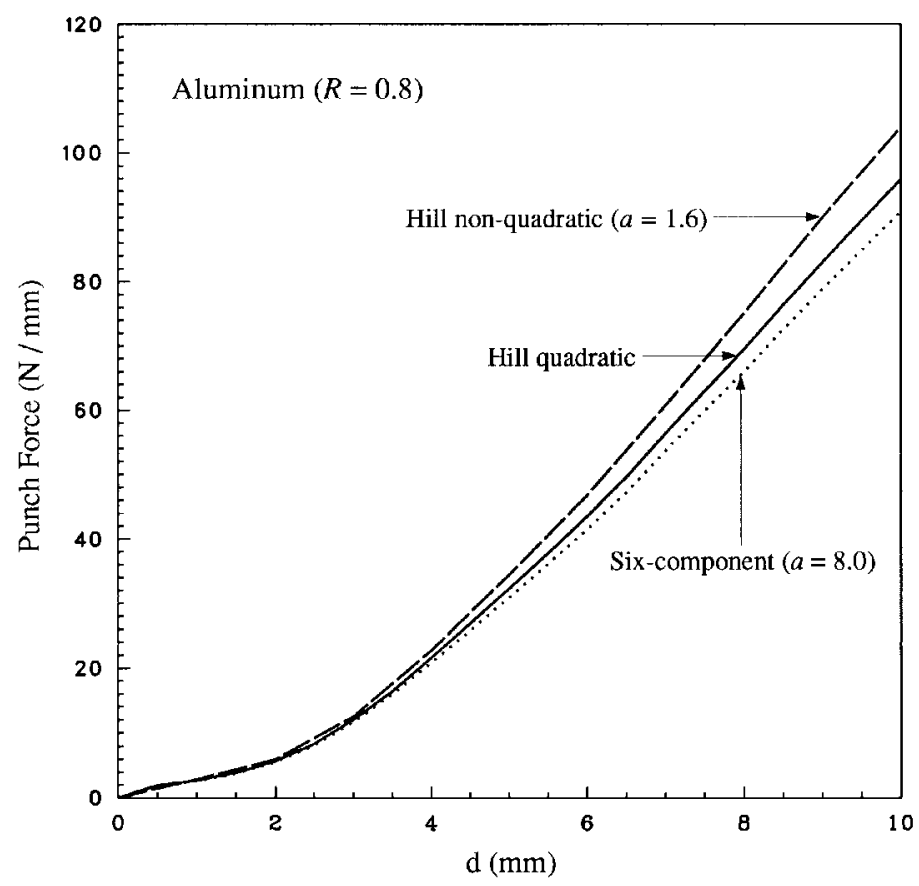

Figure 16. Comparison of the punch forces per unit width for the aluminum with $R=0 \cdot 8$ based on criterion $\mathrm{HQ}$, HNQ, and $6 \mathrm{C}$ under the plane strain drawing operation

\section{CONCLUSIONS}

The effects of the yield surface shapes based on three yield criteria on the strain distribution and the punch force are quite significant under the hemispherical punch stretching operation. A flat yield surface near the equal biaxial tension and plane strain tension states (or a low ratio of the plane strain yield stress to the equal biaxial yield stress) promotes earlier localization regardless of $R$. As indicated in the introduction, the plastic anisotropy parameter $R$ has significant effects on forming limit when the Hill quadratic yield criterion is used. On the other hand, when the higher exponent Hosford yield criterion is used, $R$ has no effect on forming limit. As shown by our computational results for hemispherical stretching operations, for a given $R$, the strain distribution and consequently the failure, depend significantly upon the yield function. Therefore, we conclude that computational simulations of forming process depend upon a good, accurate description of yielding behaviour. Using a single anisotropy parameter $R$ and a specific yield function to judge the formability of sheet metals under complex forming process can be potentially misleading.

The strain distribution and the punch force are not very sensitive to the yield criteria selected under the plane strain drawing operation. Under plane strain drawing operations, the surface strain is much larger than the average thickness strain for the critical element. This indicates the importance of investigating the effects of combined stretching and bending including loading and unloading on strain localization and failure. 


\section{APPENDIX: DETERMINATION OF ANISOTROPIC CONSTANTS FOR THE SIX-COMPONENT YIELD CRITERION}

We consider a planar isotropic sheet metal where the rolling and transverse directions are denoted as direction 1 and 2, respectively. Three constants $c_{1}, c_{2}$ and $c_{3}$ of the six-component yield criterion for planar isotropy and normal anisotropy are determined by using the yield stress under the inplane uniaxial tensile loading, the plastic anisotropy parameter $R$, and the condition of $c_{1}=c_{2}$. Implementing the uniaxial yield stress in direction 1 into equations (22)-(30) gives the following set of equations:

$$
\left|2 c_{3}+c_{2}\right|^{a}+\left|c_{2}-c_{3}\right|^{a}+\left|2 c_{2}+c_{3}\right|^{a}-2(3)^{a}=0
$$

Note that $x_{1}, x_{2}$ and $x_{3}$ in equations (26) and (27), under in-plane uniaxial loading condition in direction 1 , are expressed as

$$
x_{1}=\frac{2 \sigma_{1}}{3}, \quad x_{2}=\frac{-\sigma_{1}}{3}, \quad x_{3}=\frac{-\sigma_{1}}{3}
$$

The plastic anisotropy parameter $R$ corresponding to direction 1 can be expressed in terms of the flow rule

$$
R=\frac{\dot{\varepsilon}_{2}^{\mathrm{p}}}{-\dot{\varepsilon}_{1}^{\mathrm{p}}-\dot{\varepsilon}_{2}^{\mathrm{p}}}=\frac{\partial \Phi / \partial \sigma_{2}}{-\partial \Phi / \partial \sigma_{1}-\partial \Phi / \partial \sigma_{2}}
$$

where $\Phi$ is the six-component yield criterion in equation (22). Then $c_{1}, c_{2}$ and $c_{3}$ can be solved via equations (52) and (54) as well as condition $c_{1}=c_{2}$ by using a Newton-Raphson numerical scheme.

\section{ACKNOWLEDGEMENTS}

The authors acknowledge the support of this work by the National Science Foundation under grant number DDM-9102424 and the University Research Program of Ford Motor Company.

\section{REFERENCES}

1. N. M. Wang and B. Budiansky, 'Analysis of sheet metal stamping by a finite-element method', J. Appl. Mech., 45, 73-82 (1978).

2. S. C. Tang, 'Large elasto-plastic strain analysis of flanged hole forming', Comp. Struct., 13, 363-370 (1981).

3. N. M. Wang and S. C. Tang, 'Analysis of bending effects in sheet forming operations', Int. J. Numer. Meth. Engng., 25, 253-267 (1988).

4. A. S. Wifi, 'An incremental complete solution of the stretch forming and deep drawing of a circular blank using a hemispherical punch', Int. J. Mech. Sci., 18, 23-31 (1976).

5. B. S. Anderson, 'A numerical study of the deep drawing processes', in J. F. T. Pittman et al. (eds.), Proc. Numer. Methods in Ind. Forming Processes, Pineridge, Swansea, 1982, pp. 709-721.

6. N. Triantafyllidis and S. K. Samanta, 'Bending effects on flow localization in metallic sheets', Proc. R. Soc. Lond., A406, 205-226 (1986).

7. W. F. Hosford, 'On the crystallographic basis of yield criteria', Textures Microstructures, 26-27, 479-493 (1996).

8. R. Hill, The Mathematical Theory of Plasticity, Oxford University Press, London, 1950.

9. R. Hill, 'Theoretical plasticity of textured aggregates', Math. Proc. Camb. Phil. Soc., 85, 179-191 (1979).

10. R. H. Wagoner, J. K. Lee and G. L. Kinzel, Proc. 3rd Int. Conf. Numerical Simulation of 3-D Sheet Forming Processes, Dearborn, MI, September 29-October 3, 1996.

11. F. Barlat, D. J. Lege and J. C. Brem, 'A six-component yield function for anisotropic materials', Int. J. Plasticity, 7, 693-712 (1991). 
12. K. S. Chan, 'Effects of plastic anisotropy and yield surface shape on sheet metal stretchability', Metall. Trans., 16A, 629-639 (1985).

13. A. Marciniak and K. Kuczynski, 'Limit strains in the process of stretch forming sheet metal', Int. J. Mech. Sci., 9, 609-620 (1967).

14. D. C. Drucker, 'Relation of experiments of mathematical theories of plasticity', J. Appl. Mech., 16, 349-357 (1949).

15. F. Barlat, 'Crystallographic texture, anisotropic yield surfaces and forming limits of sheet metals', Mater. Sci. Engng., 91, 55-72 (1987).

16. W. F. Hosford, 'On yield loci of anisotropic cubic metals', Proc. 7th North Amer. Metalworking Res. Conf., SME, Dearborn, MI, 1979, pp. 191-196.

17. D. Zhou and J. Lian, 'Application of Hill's new yield theory to sheet metal forming-Part II. A numerical study of hydrostatic bulging using Hill's 1979 yield criterion', Int. J. Mech. Sci., 31, 249-263 (1989).

18. A. Graf and W. F. Hosford, 'Calculations of forming limit diagrams', Metall. Trans., 21A, 87-94 (1990).

19. J. W. Hutchinson and K. W. Neale, 'Sheet necking - III. Strain-rate effects', in D. P. Koistinen and N. M. Wang (eds.), Mechanics of Sheet Metal Forming, Plenum Press, New York, 1978, pp. 269-285.

20. K. W. Neale and E. Chater, 'Limit strain predictions for strain-rate sensitive anisotropic sheets', Int. J. Mech. Sci., 22, 563-579 (1980).

21. N. M. Wang and M. L. Wenner, 'Elastic-viscoplastic analyses of simple stretch forming problems', in D. P. Koistinen and N. M. Wang (eds.), Mechanics of Sheet Metal Forming, Plenum Press, New York, 1978, pp. 367-402.

22. D. Peirce, C. F. Shih and A. Needleman, 'A tangent modulus method for rate dependent solids', Comp. Struct., 18, $875-887$ (1984).

23. J. Lian, D. Zhou and B. Baudelet, 'Application of Hill's new yield theory to sheet metal forming — Part I. Hill's 1979 criterion and its application to predicting sheet forming limit', Int. J. Mech. Sci., 31, 237-247 (1987).

24. B. Budiansky, 'Remarks on theories of solid and structural mechanics', in Problems of Hydrodynamics and Continuum Mechanics, SIAM, Philadelphia, 1969, pp. 77-83.

25. J. W. Hutchinson, 'Finite strain analysis of elastic-plastic solids and structures', in R. F. Hartung (ed.), Numerical Solutions of Nonlinear Structural Problems, AMD, 6, ASME, New York, 1973, pp. 17-30.

26. A. K. Ghosh, 'The effect of lateral drawing-in on stretch formability', Met. Engng. Quart., 15, 53-64 (1975).

27. A. K. Ghosh and S. S. Hecker, 'Failure in thin sheets stretched over rigid punches', Metall. Trans., 6A, 1065-1074 (1975).

28. K.-C. Liao, J. Pan and S. C. Tang, 'Approximate yield criteria for anisotropic porous ductile sheet metals', Mech. Mater., in press.

29. R. W. Logan and W. F. Hosford, 'Upper-bound anisotropic yield locus calculations assuming $\langle 111\rangle$-pencil glide', Int. J. Mech. Sci., 22, 419-430 (1980). 